\title{
Optimal Control of Spraying and Drying Temperature in Production- Line based on Active Disturbance Rejection Control Technique
}

\author{
Shengyong Lei ${ }^{*}$ \\ School of Electronic Technology, Liuzhou Railway Vocational Technical College, Liuzhou, 545616, China
}

\begin{abstract}
The problems of large time delay, high inertia, and parameter uncertainty of the spraying and drying temperature control system in the automatic production line can easily lead to the instability of the system and the deterioration of anti-interference ability. Therefore, a control method based on the active disturbance rejection control (ADRC) is presented to improve the temperature control performance of the spraying and drying process in the production line. In this paper, a nonlinear ADRC temperature controller is designed, consisting of the tracking-differentiator (TD), the extended state observer (ESO), and the non-linear state error feedback (NLSEF). The ADRC controller does not rely on accurate spraying and drying process models, and it can solve the problem that the spraying and drying temperature is affected by various internal or external disturbance factors on the controlled system. Therefore, the temperature control performance of the system is improved. Simulation results show that compared with the traditional PID controller, the designed ADRC controller achieves high performance in dynamic performance, robustness, and disturbance rejection.
\end{abstract}

Keywords: active disturbance rejection control (ADRC); automatic production line; spraying and drying temperature control; large time-delay system

(Submitted on July 21, 2019; Revised on August 20, 2019; Accepted on September 23, 2019)

(C) 2019 Totem Publisher, Inc. All rights reserved.

\section{Introduction}

In the spraying and drying process of production lines including automobiles, metallurgy, and chemical engineering, the coating of workpieces contains processes including degreasing, washing, phosphating, moisture drying, primer spraying and drying, and finishing coat spraying and drying. To ensure the quality of products and save production costs, there are demanding requirements for temperature control in the spraying and drying process, generally with a temperature deviation of no more than $\pm 5{ }^{\circ} \mathrm{C}$ and hopefully within $\pm 2{ }^{\circ} \mathrm{C}$. However, there is a non-linear uncertainty relationship between the control input and output because of the nonlinearity and large time delays of the temperature control system in the spraying and drying process, apart from the possible system instability caused by large time delays. In the event of changes of the external disturbance or the control object parameter, the control effect of the conventional linear PID controller will be reduced. Therefore, it is of important practical significance to solve the temperature control problem in the production-line spraying and drying process.

At present, temperature control is still mostly performed by the traditional PID controller in the spraying and drying process of China's automatic production line [1]. The traditional PID controller can achieve a relatively satisfactory control effect for the general temperature control system, but the conventional linear PID controller has poor immunity to disturbances. The fuzzy PID control algorithm was studied and applied to the temperature control of the drying room of the automobile coating production line in [2]. Fuzzy control has certain intelligent characteristics, but the rules of fuzzy control need to be determined by experienced experts, and it is difficult to complete the complex empirical learning process in practical application. Reference [3] researched the application of the Smith estimation compensation method, the Dahlin algorithm, and the humanoid intelligent PI control algorithm in the temperature control of iron printing dryer. However, the Smith estimation compensation method and Dahlin algorithm both depend on the precise mathematical model of the controlled system, and when the controlled system changes, the control quality will deteriorate significantly. The humanoid

\footnotetext{
* Corresponding author.

E-mail address: 52003430@qq.com
} 
intelligent PI control algorithm is relatively complex, and it has a certain distance from the engineering application. The increasingly strict requirements for temperature accuracy and stability of the production process automation demand that an advanced control method more suitable for industrial control be discovered on the basis of classic PID control to obtain better control results, thus improving the product quality and saving energy.

In recent years, some positive research results have been made on the application of ADRC technology in various fields [4-14]. Active disturbance rejection control (ADRC) is a new control algorithm based on the extended state observer and the thought of eliminating error with error proposed by the famous Chinese scholar Han J. Q. in the 1990s [15-16], which inherits the "durability" and non-dependence of the mathematical model of the PID controlled system. In this paper, the technology is applied to the temperature control of the spraying and drying process in the production line. The ingenious combination of the tracking-differentiator (TD), extended state observer (ESO), and non-linear state error feedback controller (NLSEF) can contribute to the reduction of system overshooting and the maintenance of efficiency and also incorporate the external disturbance of the system into the internal total disturbance, demonstrating strong disturbance rejection ability and adaptability [17]. The simulation results verify the validity of the designed controller and obtain good control effect, which provides meaningful ideas and references for engineering applications.

The rest of this paper is organized as follows. In Section 2, the spraying and drying process in the production line is given. The principle of the spraying and drying temperature control system is addressed in Section 3. In Section 4, the design method and principle of the ADRC controller is discussed. Simulations on a large time delay and high inertia temperature control system illustrate the performances of the proposed control method in Section 5. Finally, this paper is concluded in Section 6.

\section{Spraying and Drying Process}

The automatic temperature control of the spraying and drying process is a key technology of the coating production line. For example, the coating of the automobile coating production line contains the processes of degreasing, washing, phosphating, moisture drying, primer spraying and drying, and topcoat spraying and drying, among which the demand for temperature control is more stringent in the spraying and drying processes because temperature deviations will damage product quality. Excessively high temperatures will cause pinhole or porosity on the coating surface, and excessively low temperatures will lead to insufficient curing, limpness, and small adhesion of the paint film. Spraying and drying in the automobile coating production line is generally realized through the convection drying room, whose process structure is shown in Figure 1.

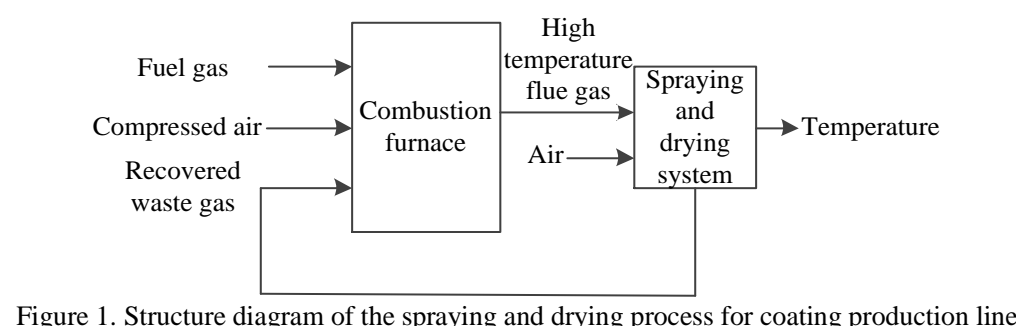

The spraying and drying system consists of the combustion chamber, drying room, heat preservation zone, electric control valve, circulating fan, and controller. The high temperature flue gas produced by the combustion furnace is sent to the drying room through the flue pipe with the circulating fan. The continuous supply of the high temperature flue gas can quickly increase the temperature of the spraying and drying room, so the temperature of the spraying and drying chamber can be regulated by adjusting the intake of high temperature flue gas. Under high temperatures, the coating and curing paint of the spraying and drying room will produce harmful gas, which may cause an explosion after reaching a certain concentration, indicating the necessity to extract the harmful gas from the spraying and drying room and send it back to the furnace for reutilization. In addition to the high temperature flue gas, the temperature in the spraying and drying room is also influenced by the atmospheric air intake, the amount of extracted exhaust gas, the size of the automotive body, and many other uncertain factors. This makes it difficult to establish a precise mathematical model, which will change due to the above factors even if it is established.

\section{The Working Principle of the Spraying and Drying Temperature Control System}

The spraying and drying temperature control system is composed of the host computer, PLC, AD module, DA module, electric control valve, temperature sensor, and temperature transmitter, as shown in Figure 2. The temperature transmitter sends the transformed signal of the detected temperature of the drying room to the PLC through the AD module. Then, the 
temperature value is sent to the host computer, where the collected temperature values are compared with the set value. Then, the best controlled quantity is obtained through analysis with the ADRC algorithm, which is sent to the DA module through the PLC, thus realizing the real-time regulation of the opening of the electric control valve and the control of the intake of high temperature flue gas and eventually regulating the temperature of the drying room.

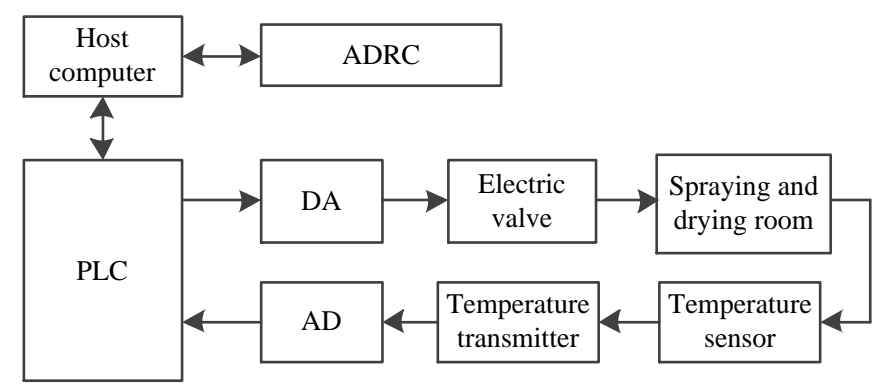

Figure 2. Composition of the spraying and drying temperature control system

\section{Design of Active Disturbance Rejection Controller}

The automatic temperature control process of spraying and drying is a system with delay and inertia. According to the temperature changes detected by the system, there is a certain time delay and inertia from the regulation of the high temperature flue gas intake to temperature changes in spraying and drying room, which can be approximately simplified as a first-order delay inertia link.

$$
G(s)=\frac{y(s)}{u(s)}=\frac{K}{T s+1} \times e^{-\tau s}
$$

Where $G(s)$ is the transfer function between the temperature of the spraying and drying chamber and the opening of the electric control valve for high temperature flue gas, $y(s)$ is the function of temperature in the spraying and drying room, $u(s)$ is the function of electric control valve opening for the high temperature flue gas, $K$ is the proportionality coefficient, $T$ is the time constant, and $\tau$ is the delay time (s).

Due to the influence of several uncertain factors on the temperature of spraying and drying, the parameters $K$ and $T$ in the simplified first-order control system will vary along with the real-time change of intake air volume, volume of extracted air, and working environment, leading to the variability of the mathematical model. Therefore, the control system cannot carry out automatic control completely relying on the mathematical model of the spraying and drying process. The development of the active disturbance rejection control technology provides a new way to deal with the systems without precise mathematical models. Active disturbance rejection control integrating the advanced control theory and the traditional PID control thinking is a new approach to solve such problems.

\subsection{The Basic Structure}

ADRC is a new type of nonlinear controller whose predecessor is the PID controller. The most important characteristic is that it inherits the advantages of the PID controller, including the non-dependence of the mathematical model of the controlled object and the adaptability in industrial applications. It avoids its disadvantages of being easy-to-overshoot [18], possibly unreasonable in linear combination, slow in system response due to error integral feedback, oscillation-prone, and saturated in controlled quantity [16]. The controller based on the active disturbance rejection control is composed of the tracking-differentiator (TD), extended state observer (ESO), and non-linear state error feedback (NLSEF). The spraying and drying temperature control system as a first-order inertial system with large delay can be processed into a second-order controlled system with the "order increasing method" [15,19], so the control system adopts the second-order active disturbance rejection controller. Its structure principle is shown in Figure 3.

In Figure 3, $v$ is the control target or given signal of the system, which is the set value of the spraying and drying temperature. It conducts the tracking of the given signal and the extraction of the differential signal through the trackingdifferentiator (TD) instead of directly calculating the output signal error of the control system. The output signals of TD are $v_{1}$ and $v_{2}$, among which the former is the tracking signal of the given signal and the latter is the differential signal of the given signal. The designed tracking differentiator better resolves the contradiction between the overshoot and quick response of the control system, and the extracted differential signal lays the foundation for the design of error feedback 
controller in the meantime. The extended-state observer (ESO) is the core part of the active disturbance rejection controller, equipped with the functions of disturbance tracking and disturbance compensation. Non-dependent of the mathematical model of the controlled system, it estimates the state and disturbance of the system with the output $y$ of the controlled system and the output $u$ of the controller. In Figure 3, the output $y$ of the controlled system and the output $u$ of the controller are taken as the input signal of the ESO to obtain the tracking signal $z_{1}$, the differential signal $z_{2}$, and the signal $z_{3}$ with disturbance compensation effect. The $b_{0}$ here is the gain of the known control amount. The error signal $e_{1}$ is formed by the tracking signal of TD and ESO, and the error signal $e_{2}$ is produced by the differential signal of TD and ESO. They are taken as the input signal of the non-linear error feedback controller (NLSEF) to output the control signal $u_{0}$ through the internal nonlinear combination. Under the common action of $u_{0}$ and $z_{3}$, we can acquire the control signal $u$ of the controller, which can be used to change the electric valve opening of the high temperature flue gas and thus realize the automatic temperature control of the spraying and drying chamber.

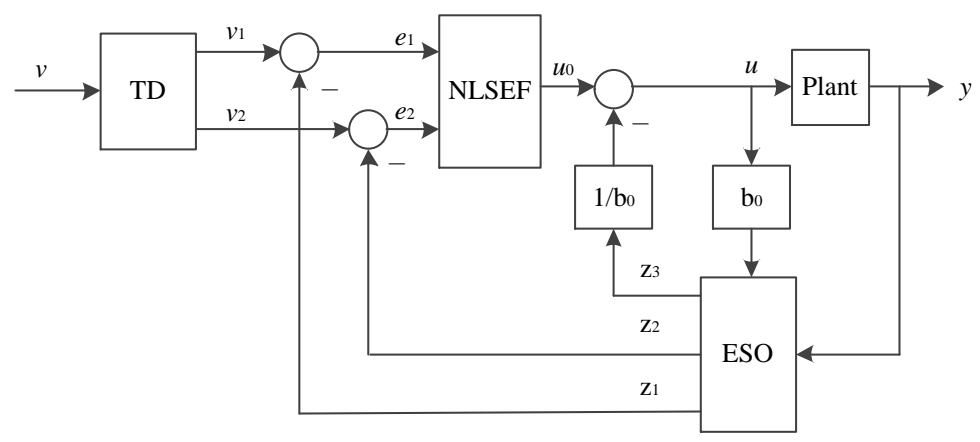

Figure 3. Structure diagram of the second-order active disturbance rejection controller

\subsection{The Control Algorithm}

To achieve the disturbance tracking and disturbance compensation control of the controlled system in real time, it is necessary to design the control algorithms of the tracking differentiator, extended state observer, and nonlinear error feedback controller [15,20], which can be designed and debugged separately before the final joint debugging.

The control algorithm for the tracking-differentiator is shown in Equation (2).

$$
\left\{\begin{array}{l}
e=v_{1}-v \\
f h=\operatorname{fhan}\left(e, v_{2}, r_{0}, h_{0}\right) \\
v_{1}=v_{1}+h v_{2} \\
v_{2}=v_{2}+h f h
\end{array}\right.
$$

Where $h$ is the simulation step size, $r_{0}$ is the velocity factor, and $h_{0}$ is the filter factor. fhan $\left(x_{1}, x_{2}, r, h\right)$ is the discrete time optimization control synthesis function [15], and the algorithm is defined as

$$
\left\{\begin{array}{l}
d=r h \\
d_{0}=h d \\
y=x_{1}+h x_{2} \\
a_{0}=\sqrt{d^{2}+8 r|y|} \\
a=\left\{\begin{array}{l}
x_{2}+\frac{\left(a_{0}-d\right)}{2} \operatorname{sign}(y),|y|>d_{0} \\
x_{2}+\frac{y}{h},|y| \leq d_{0}
\end{array}\right. \\
\text { fhan }=-\left\{\begin{array}{l}
r \operatorname{sign}(a),|a|>d \\
r \frac{a}{d},|a| \leq d
\end{array}\right.
\end{array}\right.
$$


Where $\operatorname{sign}(x)$ is a sign function.

The extended state observer (ESO) estimates the state and disturbance of the system with the output $y$ of the controlled system and the output $u$ of the controller. The control algorithm is shown in Equation (4).

$$
\left\{\begin{array}{l}
e=z_{1}-y \\
f e=f a l\left(e, \alpha_{1}, \delta\right) \\
f e_{1}=f a l\left(e, \alpha_{2}, \delta\right) \\
z_{1}=z_{1}+h\left(z_{2}-\beta_{01} e\right) \\
z_{2}=z_{2}+h\left(z_{3}-\beta_{02} f e+b_{0} u\right) \\
z_{3}=z_{3}+h\left(-\beta_{03} f e_{1}\right)
\end{array}\right.
$$

Where $\beta_{01}, \beta_{02}$, and $\beta_{03}$ are the gain adjustment parameters. The fal function is the power function, mainly used to prevent high frequency oscillation, and it is defined as

$$
f a l(e, \alpha, \delta)= \begin{cases}\frac{e}{\delta^{1-\alpha}}, & |e| \leq \delta \\ |e|^{\alpha} \operatorname{sign}(e), & |e|>\delta\end{cases}
$$

Where $\delta$ is the interval length of the linear segment and $\alpha$ is the power parameter, whose value is generally in the range of $0<\alpha \leq 1$.

The algorithm of the nonlinear state error feedback controller (LNSEF) is shown in Equation (6).

$$
\left\{\begin{array}{l}
e_{1}=v_{1}-z_{1}, e_{2}=v_{2}-z_{2} \\
u_{0}=\beta_{1} \times \operatorname{fal}\left(e_{1}, \alpha_{01}, \delta_{1}\right)+\beta_{2} \times \operatorname{fal}\left(e_{2}, \alpha_{02}, \delta_{1}\right)
\end{array}\right.
$$

In the formula, the feedback control law of the controller is in the nonlinear PD combining form. $\beta_{1}$ and $\beta_{2}$ are the error feedback gain adjustment parameters, and the fal function is defined in Equation (5). The final controlled quantity can be obtained with Figure 3 and Equation (6) as follows:

$$
u=u_{0}-\frac{z_{3}}{b_{0}}
$$

The $b_{0}$ here is the estimated gain coefficient of the controlled quantity, whose value needs to be estimated if unknown.

\subsection{Parameter Tuning}

The design of the active disturbance rejection controller of production-line spraying and drying temperature requires the determination of several parameters, mainly including the parameters of TD, ESO, and LNSEF such as TD $\left\{r_{0}, h_{0}\right\}$, ESO $\left\{\alpha_{1}, \alpha_{2}, \delta, \beta_{01}, \beta_{02}, \beta_{03}\right\}$, and LNSEF $\left\{\alpha_{01}, \alpha_{02}, \delta_{1}, \beta_{1}, \beta_{2}\right\}$ to reach a total of 13 . The parameters that need to be repeatedly debugged in the experiments are the five parameters of $\beta_{01}, \beta_{02}, \beta_{03}, \beta_{1}$, and $\beta_{2}$.

The parameter tuning of TD $\left\{r_{0}, h_{0}\right\}: r_{0}$ is the velocity factor for the tracking differentiator. A greater $r_{0}$ indicates a shorter time for the tracking signal to reach the given value, and a smaller one means longer time. Generally, there is a relation between $r_{0}$ and the bandwidth of TD as follows [15]:

$$
r_{0}=\frac{\omega_{0}^{2}}{1.14}
$$

Where $\omega_{0}$ is the bandwidth of TD.

The other parameter $h_{0}$ of TD is a filter factor, and $h$ is the integration step; we generally take $h_{0}=k h, k=1,2, \cdots, n$. 
The smaller integration step is more conducive to the realization of signal tracking and noise suppression, but a much smaller value of $h$ will lead to the oscillation of the differential signal; thus, the integration step of TD needs to be amplified by $k$ times during the resistance to differential noise.

Parameters tuning of ESO $\left\{\alpha_{1}, \alpha_{2}, \delta, \beta_{01}, \beta_{02}, \beta_{03}\right\}: \alpha_{1}$ and $\alpha_{2}$ determine the type of the power function. To improve the efficiency of parameter estimation, ESO is determined to be a non-linear function with $\alpha_{1}=0.5$ and $\alpha_{2}=0.25$. A large $\delta$ value indicates a long linear work region and poor performance of ESO, and a small $\delta$ value may cause high frequency oscillation. Therefore, we generally take $\delta=h$. The three parameters of $\beta_{01}, \beta_{02}$, and $\beta_{03}$ are the gain parameters of ESO, which have relatively great influence on the working performance of ESO. They can be debugged according to the following formula in the initial debugging [15]:

$$
\beta_{01}=\frac{1}{h}, \beta_{02}=\frac{1}{3 h^{2}}, \beta_{03}=\frac{1}{20 h^{3}}
$$

Where $h$ is the integration step. In general, there is a gap of a magnitude order between $\beta_{01}, \beta_{02}$, and $\beta_{03}$. In the actual application, they need to be repeatedly debugged in accordance with the control effect based on Equation (9) to finally determine the appropriate parameter values.

Parameter tuning of LNSEF $\left\{\alpha_{01}, \alpha_{02}, \delta_{1}, \beta_{1}, \beta_{2}\right\}$ : the three parameters of $\alpha_{01}, \alpha_{02}$, and $\delta_{1}$ can be debugged with reference to the parameters of ESO to obtain $\alpha_{01}=0.75, \alpha_{02}=1.5$, and $\delta_{1}=h . \beta_{1}$ and $\beta_{2}$ can be debugged according to the proportionality coefficient and the deferential coefficient of PD controller.

\section{Simulation Experiment and Analysis}

To verify the effectiveness of the active disturbance rejection controller, a simulation test is conducted by taking the spraying and drying temperature control system of a production line as an example. The mathematical model of the system is approximated to

$$
G(s)=\frac{12.5}{1200 s+1} e^{-350 s}
$$

In the formula, the time constant $T$ of the system is 1,200 seconds and the lag time $\tau$ is 350 seconds, indicating a typical system with high inertia and large time delays. The time constant of the system may change along with the working condition changes during the operating process.

In the experimental simulation platform of matlab7.0, the simulation control test is conducted on the spraying and drying temperature control system. The parameter values of the active disturbance rejection controller are $h=0.1, r_{0}=1, h_{0}$ $=1, \alpha_{1}=0.5, \alpha_{2}=0.25, \delta=0.1, \beta_{01}=0.8, \beta_{02}=100, \beta_{03}=76, \alpha_{01}=0.75, \alpha_{02}=1.5, \delta_{1}=0.1, \beta_{01}=10$, and $\beta_{02}=0.3$. Meanwhile, the PID control commonly used in industry is selected to complete the comparison of the control effects. The proportion, integration, and deferential parameters of PID obtained through several experiments are $K_{p}=0.17, K_{i}=0.000175$, and $K_{d}=$ 0.001. The sampling time of the two kinds of controllers is 0.1 seconds.

\subsection{Dynamic Performance Analysis}

The system heats up from $21^{\circ} \mathrm{C}$ after a 350 -second delay with a control objective of $180^{\circ} \mathrm{C}$. The response curves of ADRC and PID are shown in Figure 4. The PID control process demonstrates significant overshoot (a maximum of 22\%) and becomes stable after regulating two oscillations for about 3,400 seconds. The ADRC control reaches the steady state after a 2,200-second adjustment without occurrence of overshoot. ADRC, which is characterized by short response time, no overshoot, and stable control, has significantly better dynamic performance than PID control.

\subsection{Robust Performance Analysis}

In verifying the robustness of the active disturbance rejection control, the time constant of the system will be changed when the stimulated working conditions of the spraying and drying process are different. Therefore, in the case of constant controller parameters, the ADRC and PID controllers are employed to carry out the control experiments when the time constant $\mathrm{T}$ of the system is 1,200 seconds, 1,400 seconds, and 1,000 seconds. The ADRC controller has excellent control effects, as shown in Figure 5. 


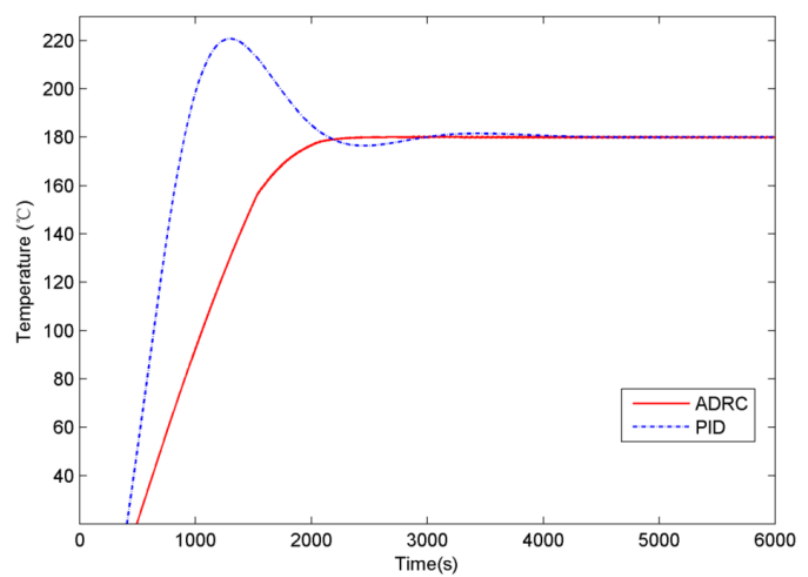

Figure 4. The control effect comparison of ADRC and PID
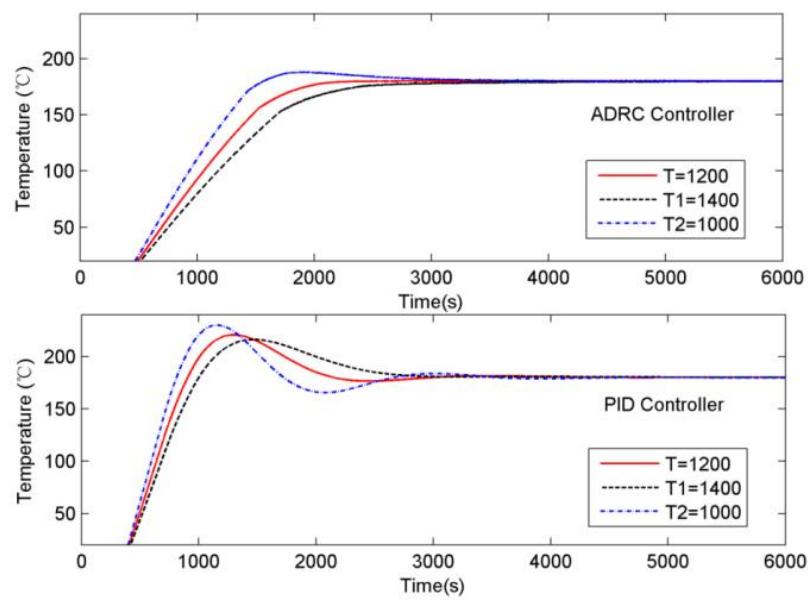

Figure 5. Response curves of ADRC and PID for the three different transfer functions

\subsection{Disturbance Rejection Analysis}

To verify the immunity of the ADRC controller, we send an interfering sinusoidal signal with an amplitude value of 10 and a cycle of 500 seconds into the feedback channel of the control system. The control of ADRC is still relatively stable without being influenced by the disturbance. Meanwhile, the PID control is affected to a relatively great extent with a $\pm 5 \%$ amplitude fluctuation of the output signal, as shown in Figure 6. This shows that ADRC possesses stronger disturbance rejection capability and a more stable control effect than PID control.
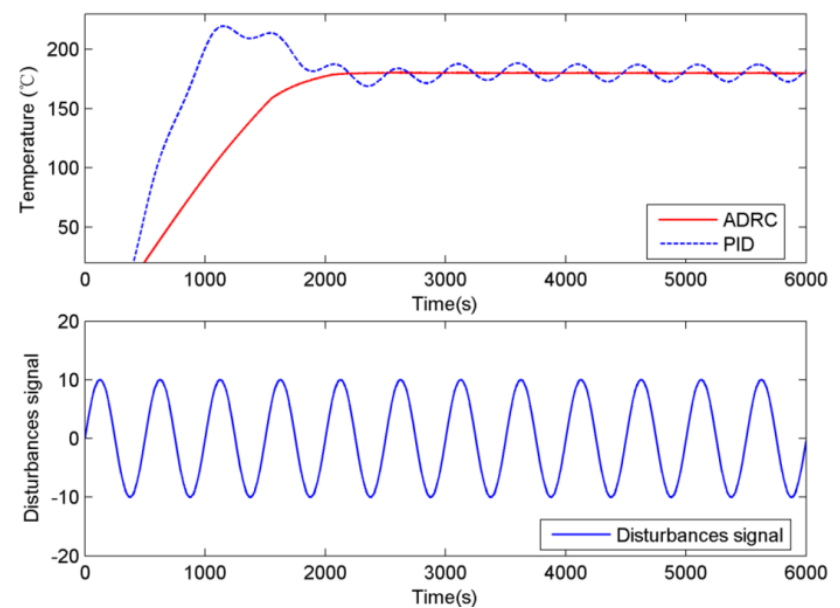

Figure 6. Response curves of ADRC and PID with low frequency sinusoidal interference signal 


\section{Conclusions}

In this paper, the temperature control strategy based on the second-order nonlinear active disturbance rejection technology is applied to the temperature control of the spraying and drying production line characterized by high inertia, large time delays, and time variance.

(1) Compared with the traditional PID control, the proposed active disturbance rejection control strategy has better control effects. The experimental results demonstrate that a high performance is obtained with stable regulation, little overshoot, and strong disturbance rejection ability. Moreover, the system does not rely on an exact mathematical model and has good adaptability to working conditions.

(2) The proposed active disturbance rejection control system is distinct in structure, with simple control algorithms, and suitable for engineering applications. Before its applications to projects, the real-time control can be simulated on the experimental system of the spraying and drying production line for the debugging of relevant parameters according to the running conditions of the system.

\section{Acknowledgements}

This work is supported by the Basic Ability Promotion Project for Young and Middle-aged Teachers in Guangxi Colleges and Universities of China (No. KY2016YB761).

\section{References}

1. D. M. Zhang, "Temperature Control and Realization of Spraying and Drying Production Line based on PLC," M.S. Thesis, Northeastern University, Shenyang, China, 2011

2. B. Wu, "Research and Design of Control System of Drying Room in Automobile Coating Production Line," M.S. Thesis, Jiangsu University, Zhenjiang, China, 2016

3. S. H. Wang, "Research on Control Technologies for Iron Printing Chamber Product Line Automatic System," Ph.D. Dissertation, Nanjing University of Science and Technology, Nanjing, China, 2007

4. B. Y. Liu, C. A. Zhu, and X. Z. Guo, "Current-Loop Control for the Pitching Axis of Aerial Cameras via an Improved ADRC," Mathematical Problems in Engineering, Vol. 2017, pp. 1-8, 2017

5. F. Fang, R. L. Li, and J. Z. Liu, "Active Disturbance Rejection Control for Bed Temperature of Circulating Fluidized Bed Boiler based on Multivariable Internal Model Structure," Proceedings of the CSEE, Vol. 37, No. 1, pp. 172-180, 2017

6. B. Zhang and H. B. Gao, "Active Disturbance Rejection Control Algorithm Research for Large Tracking Dome System," Science Technology and Engineering, Vol. 16, No. 26, pp. 75-79, 2016

7. D. Wu, T. Zhao, and K. Chen, "Research and Industrial Applications of Active Disturbance Rejection Control to Fast Tool Servos," Control Theory and Applications, Vol. 30, No. 12, pp. 1354-1362, 2013

8. L. M. Hou, M. J. Zhang, W. Wang, and X. J. Li, "Sliding Mode Control Speed Modulation System for PMSM based on ADRC," Small and Special Electrical Machines, Vol. 39, No. 4, pp. 50-52, 2011

9. L. J. Wang, C. N. Tong, Q. Li, Y. X. Yin, Z. Q. Gao, and Q. L. Zheng, "Practical Active Disturbance Rejection Solution for Monitoring Automatic Gauge Control System with Large Time-Delay," Control Theory and Applications, Vol. 29, No. 3, pp. $368-374,2012$

10. L. Zhao, Y. F. Yang, Y. Q. Xia, and Z. X. Liu, "Active Disturbance Rejection Position Control for a Magnetic Rodless Pneumatic Cylinder," IEEE Transactions on Industrial Electronics, Vol. 62, No. 9, pp. 1-8, 2015

11. R. Q. Shi, J. M. Song, and W. Y. Wan, "Active Disturbance Rejection Control System Design for a Morphing Wing Structure," Asian Journal of Control, Vol. 17, No. 3, pp. 832-841, 2015

12. G. F. Sun, X. M. Ren, and D. W. Li, "Neural Active Disturbance Rejection Output Control of Multimotor Servomechanism," IEEE Transactions on Control Systems Technology, Vol. 23, No. 2, pp. 746-753, 2015

13. J. Tao, Q. L. Sun, Z. Q. Chen, and Y. P. He, "Autonomous Homing Control of a Parafoil System based on ADRC," in Proceedings of 2016 IEEE Chinese Guidance, Navigation and Control Conference, pp. 110-115, 2016

14. J. M. Liu, Y. W. Sun, C. Fu, and Y. G. Li, "Battery Energy Storage System based on Power Electronic Transformer and Its ADRC Control Strategy," High Voltage Engineering, Vol. 43, No. 1, pp. 131-140, 2017

15. J. Q. Han, "Active Disturbance Rejection Control Technique - the Technique for Estimating and Compensating the Uncertainties," National Defense Industry Press, pp. 243-351, Beijing, 2008

16. J. Q. Han, "From PID to Active Disturbances Rejection Control," IEEE Transactions on Industrial Electronics, Vol. 56, No. 3, pp. $900-906,2009$

17. J. Li, X. H. Qi, H. Wan, and Y. Q. Xia, "Active Disturbance Rejection Control: Theoretical Results Summary and Future Researches," Control Theory and Applications, Vol. 34, No. 3, pp. 1-15, 2017

18. G. P. Teppa and G. Garcia, "Design of an Optimal PID Controller for a Coupled Tanks System Employing ADRC," IEEE Latin America Transactions, Vol. 15, No. 2, pp. 189-196, 2017

19. L. J. Wang, Q. Li, C. N. Tong, and Y. X. Yin, "Overview of Active Disturbance Rejection Control for Systems with TimeDelay," Control Theory and Applications, Vol. 30, No. 12, pp. 1521-1533, 2013 
20. Y. Q. Xia, L. Dai, M. Y. Fu, C. M. Li, and C. M. Wang, “Application of Active Disturbance Rejection Control in Tank Gun Control System," Journal of the Franklin Institute, Vol. 351, No. 4, pp. 2299-2314, 2014

Shengyong Lei received his B.S. degree in applied electronic technology from China Agricultural University in 2001 and his M.S. degree in control theory and control engineering from Guangxi University in 2008. Currently, he is an associate professor and senior engineer at Liuzhou Railway Vocational Technical College. 